\title{
How Flies Fly
}

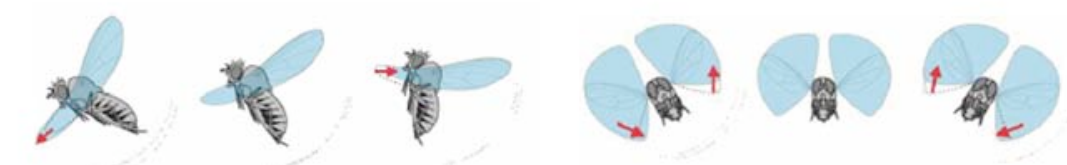

\section{Michael Dickinson}

California Institute of Technology, Pasadena, CA

Like all forms of locomotion, flight behavior results from a complex set of interactions, not just within circuits in the brain, but among neurons, muscles, skeletal elements, and physical process within the external world. To control flight, the fly's nervous system must generate a code of motor information that plays out through a small but complicated set of power and steering muscles. These muscles induce microscopic oscillations in an external skeleton that drive the wings back and forth 200 times each second producing a time-variant pattern of aerodynamic forces that the fly modulates to steer and maneuver through the air. The animal's motion through space alters the stream of information that runs through an array of visual, chemical, and mechanical sensors, which collectively provide feedback to stabilize flight and orient the animal towards specific targets. The goal of the research in my laboratory is to 'reverse engineer' this flight control system, and thus determine the means by which the nervous system controls the animal's trajectory through space. 


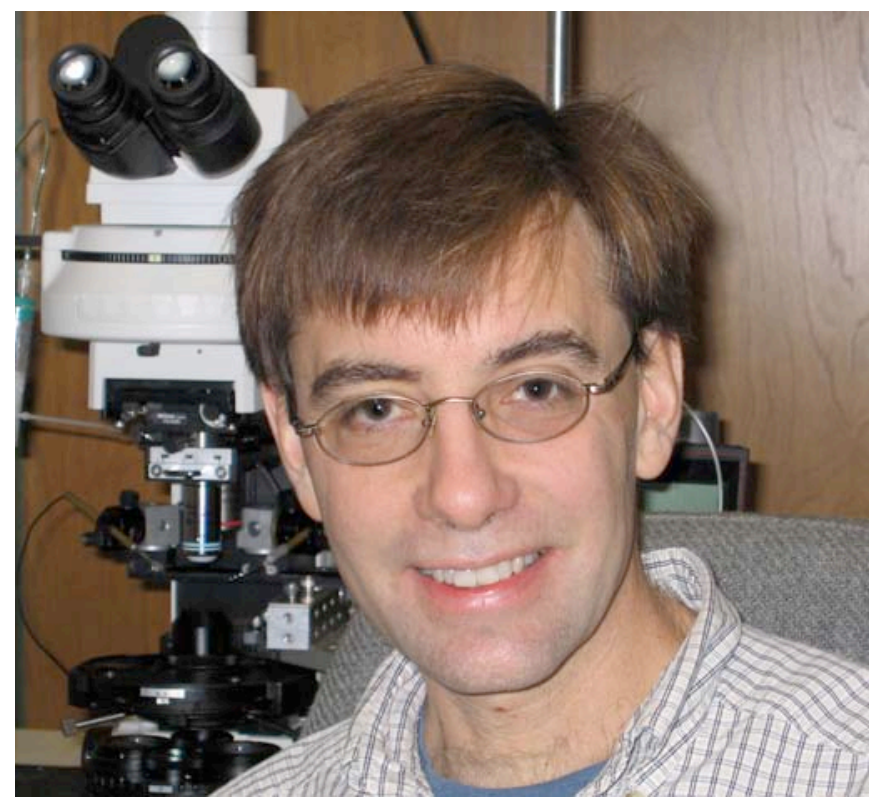

Michael Dickinson graduated from Brown University in 1984, with a B.S. in Neuroscience. He received his Ph. D. from the University of Washington 1989 in the Dept. of Zoology. His dissertation research focused on the development and physiology of tiny sensory structures on the wings of flies. As a post-doctoral researcher, he worked briefly at the Max Planck Institute for Biological Cybernetics in Tübingen, Germany, and served as an Assistant Professor in the Dept. of Anatomy at the University of Chicago in 1991. He moved to University of California, Berkeley in 1996 and was appointed as the Williams Professor in the Department of Integrative Biology in 2000. He moved to Caltech in 2002, and is currently the Esther and Abe Zarem Professor of Bioengineering.

Dr. Dickinson's research focuses on the mechanics and physiology of animal behavior. Specifically, he has studied the flight behavior of insects simultaneously at several levels of analysis, in an attempt to integrate cellular physiology, biomechanics, aerodynamics, and behavior. His awards include the Larry Sandler Award from the Genetics Society of America (1990), a Packard Foundation Fellowship in Science and Engineering (1992), the Quantrell award for Excellence in Undergraduate Teaching (1993), and the George A. Bartholemew Award for Comparative Physiology from the American Society of Zoologists (1995). In 2001, he was awarded and a MacArthur Foundation Fellowship.

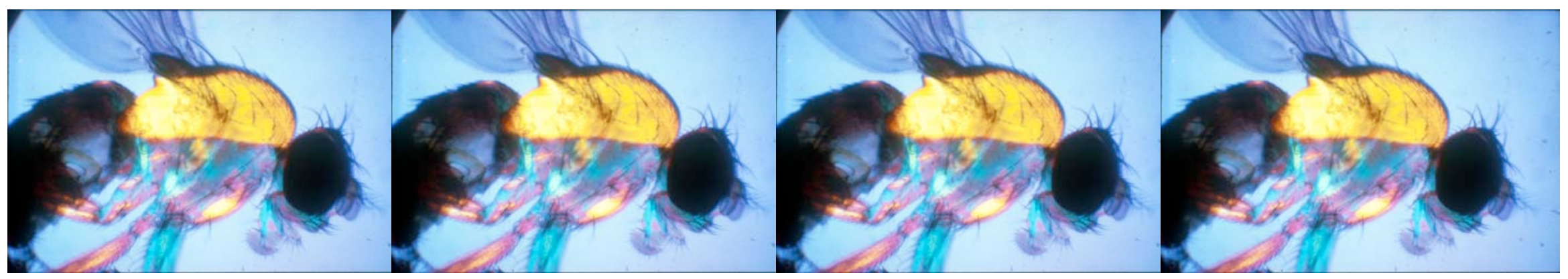

